\title{
MOCCA - A system that learns and recommends visual preferences based on cultural similarity
}

\author{
Reinecke, Katharina ; Minder, Patrick ; Bernstein, Abraham
}

\begin{abstract}
We demonstrate our culturally adaptive system MOCCA, which is able to automatically adapt its visual appearance to the user's national culture. Rather than only adapting to one nationality, MOCCA takes into account a person's current and previous countries of residences, and uses this information to calculate user-specific preferences. In addition, the system is able to learn new, and refine existing adaptation rules from users' manual modifications of the user interface based on a collaborative filtering mechanism, and from observing the user's interaction with the interface.
\end{abstract}

DOI: https://doi.org/10.1145/1943403.1943495

Posted at the Zurich Open Repository and Archive, University of Zurich

ZORA URL: https://doi.org/10.5167/uzh-63242

Conference or Workshop Item

Published Version

Originally published at:

Reinecke, Katharina; Minder, Patrick; Bernstein, Abraham (2011). MOCCA - A system that learns and recommends visual preferences based on cultural similarity. In: 16th International Conference on Intelligent User Interfaces (IUI), Palo Alto, 13 February 2011 - 16 February 2011. ACM, 453-454.

DOI: https://doi.org/10.1145/1943403.1943495 


\section{MOCCA - A System That Learns and Recommends Visual Preferences Based on Cultural Similarity}

\author{
Katharina Reinecke \\ Department of Informatics \\ University of Zurich \\ Switzerland \\ reinecke@ifi.uzh.ch
}

\author{
Patrick Minder \\ Department of Informatics \\ University of Zurich \\ Switzerland \\ minder@ifi.uzh.ch
}

\author{
Abraham Bernstein \\ Department of Informatics \\ University of Zurich \\ Switzerland \\ bernstein@ifi.uzh.ch
}

\begin{abstract}
We demonstrate our culturally adaptive system MOCCA, which is able to automatically adapt its visual appearance to the user's national culture. Rather than only adapting to one nationality, MOCCA takes into account a person's current and previous countries of residences, and uses this information to calculate user-specific preferences. In addition, the system is able to learn new, and refine existing adaptation rules from users' manual modifications of the user interface based on a collaborative filtering mechanism, and from observing the user's interaction with the interface.
\end{abstract}

\section{Author Keywords}

Cultural Similarity, Cultural Adaptivity, User Interface Design, Localization

\section{ACM Classification Keywords}

H.5.2 Information Interfaces and Presentation: User Interfaces

\section{General Terms \\ Human Factors}

\section{INTRODUCTION}

Website designs are a matter of taste. Websites that match our visual preferences are perceived as much more trustworthy, intuitive, and appealing $[4,3]$. Those that do not, miss the chance that we return. However, what we consider as "good design" is controlled by various outside influences, which teach us conventions, values, and beliefs. We call these influences "culture".

Our culture determines our preference for certain colors, whether we like blinking animations, or no images at all, or if we think that web sites should be structured clearly in order to convey credibility. Moreover, culture also influences perceptional abilities. It dictates the amount of unstructured information we can absorb at once [1], where we place our focus of attention first [2], or how easily we develop a "lost

Copyright is held by the author/owner(s).

IUI'11, February 13-16, 2011, Palo Alto, California, USA. ACM 978-1-4503-0419-1/11/02. in hyperspace" feeling in a non-linear environment [1]. Our culturally adaptive web application MOCCA knows your preferences and capabilities. MOCCA is designed to increase user satisfaction by automatically adapting its interface to cultural preferences.

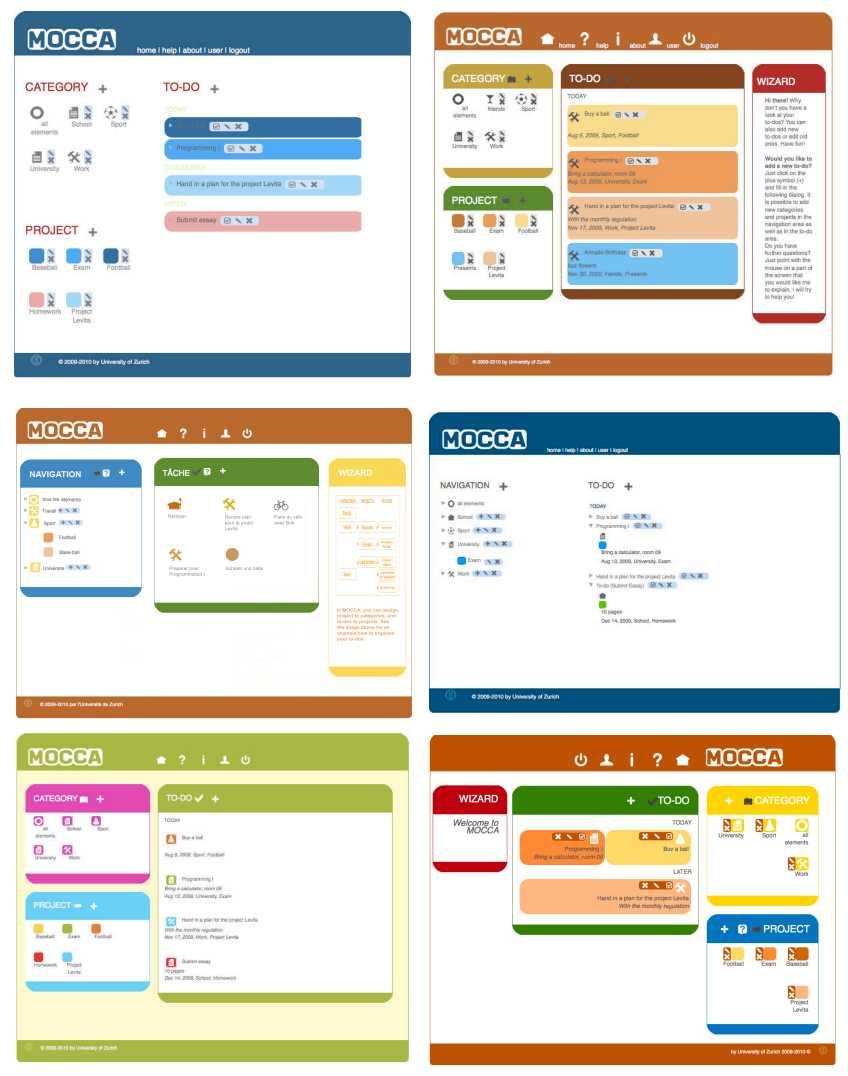

Figure 1. Example interfaces of MOCCA

\section{SYSTEM OVERVIEW}

MOCCA (see Figure 1 for some example interfaces) is a web-based to-do list application, developed to illustrate an approach to cultural adaptivity. MOCCA takes as input your current country of residence and possible former countries of residence in order to create a personalized interface. Depending on how long you've lived in different places, it calculates your personal cultural background, links it to the corresponding preferences, and modifies its interface accord- 
ingly. Of course, MOCCA is not always right. It therefore incorporates two types of learning possibilities:

- User interaction tracking: MOCCA infers new adaptations by observing the user's mouse movements and key strokes. Clustering several users into different levels of computer literacy, it re-estimates a person's abilities, and triggers new adaptations, such as an increase in support, a reduction of the number of functionalities, or a different content structuring.

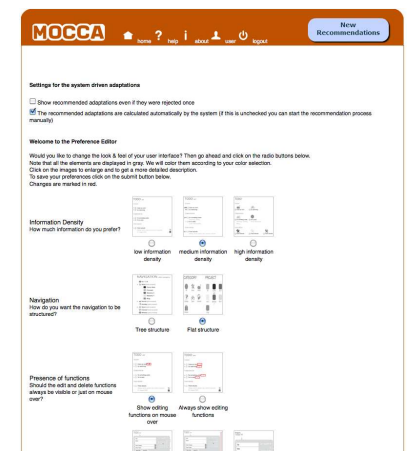

Figure 2. MOCCA's preference editor

- Recommendation mechanism: Users have the ability to manually change their interface in a preference editor (Figure 2). Based on the assumption that people of similar cultural background often share similar preferences, MOCCA uses the choices of all registered users to improve the predictions for new users, and to recommend adaptations to existing users. Thus, its ability to provide suitable user interfaces improves over time.

\section{Beyond Localization}

While many international companies adapt their websites to the design requirements of specific countries, this localization process is usually restricted to a modification of language, or date and time formats. In contrast, MOCCA provides an extensive re-arrangement of its interface in order to provide a personalized look \& feel. The adaptations include visual aspects, such as colorfulness, brightness and contrast of colors, the complexity, structure, and image-to-text ratio. Beyond this, MOCCA also adapts workflow-related aspects, such as the navigation, the number of functionalities available, the level of guidance through dialogs, or the general support. With more than a $115^{\prime} 000$ different combination possibilities of its interface elements, it is much superior over conventional localization efforts.

The system also takes into account possible migrational backgrounds. In conventional approaches to localization users are either required to decide for one country (e.g., by clicking on a flag), or they are automatically presented a localized web site based on their IP address. Exceeding this restriction, MOCCA also provides suitable interfaces for users who have lived in (and are influenced by) more than one country, or who are temporarily located in a country that does not represent their national culture.

\section{Technical Background}

In MOCCA, user interface elements are developed as Java Server Pages (JSP), which can be loaded and compiled by an Apache Tomcat server at runtime. The use of AJAX (Asynchronous Javascript and XML) allows communication between the browser and server, without the need for a whole page to be reloaded. To store and query information about the user's culture, MOCCA is connected to an applicationindependent cultural user model ontology [6]. In addition, an adaptation ontology holds all of its user interface elements and the corresponding rules, which determine the required adaptations. The adaptation ontology also specifies certain restrictions for each element, such as their interdependence (e.g. element A can only appear on the user interface in combination with element B), and it defines an element's possible placement areas within the user interface, as well as its minimum and maximum size.

\section{FIRST RESULTS AND FUTURE WORK}

In a previous user test with 30 international users, MOCCA has demonstrated its ability to predict the majority of user preferences [5], thus, showing that it is possible to automatically generate suitable interfaces based on the information about a person's current and former countries of residence. In the future, we intend to conduct a larger study in order to evaluate MOCCA's accuracy of prediction and recommending visual preferences after learning from users' feedback over a certain amount of time.

\section{ACKNOWLEDGEMENTS}

We thank the Hasler Foundation for partly funding this research under research grant no. 2322.

\section{REFERENCES}

1. I. Burgmann, P. Kitchen, and R. Williams. Does Culture Matter on the Web? Marketing Intelligence \& Planning, 24(1):62-73, 2006.

2. T. Chan and B. Bergen. Writing Direction Influences Spatial Cognition. In Annual Conference of the Cognitive Science Society, 2005.

3. B. Corbitt, T. Thanasankit, and J. Haynes. A Model for Culturally-Informed Web Interfaces. In Internet Management Issues: A Global Perspective, pages 1-26. IGI Global, 2002.

4. D. Cyr, C. Bonanni, and J. Ilsever. Design and e-Loyalty Across Cultures in Electronic Commerce. In Proceedings of the 6th International Conference on Electronic Commerce, 2004.

5. K. Reinecke and A. Bernstein. Tell Me Where You've Lived, and I'll Tell You What You Like: Adapting Interfaces to Cultural Preferences. In International Conference on User Modeling, Adaptation, and Personalization (UMAP), 2009.

6. K. Reinecke, G. Reif, and A. Bernstein. Cultural User Modeling With CUMO: An Approach to Overcome the Personalization Bootstrapping Problem. In Workshop on Cultural Heritage on the Semantic Web, International Semantic Web Conference, 2007. 